\title{
Efficacité comparée de la pollinisation d'espèces sauvages d'Helianthus par plusieurs genres d'insectes
}

\author{
S Meynié *, R Bernard \\ Unité de formation et de recherches génétique et amélioration des plantes, Inra-Montpellier, domaine de Melgueil, \\ F-34130 Mauguio, France
}

(Reçu le 4 juillet 1996 ; accepté le 5 novembre 1996)

\begin{abstract}
Summary - Pollinator efficiency of some insects in relation to wild species populations of Helianthus L. The efficiency of four insect species (Apis mellifera, Bombus terrestris, Megachile rotundata and the Calliphora spp flies) in pollinating three wild sunflower species ( $H$ annuus, $H$ argophyllus, $H$ debilis ssp cucumerifolius) in insect-proof cages was compared with hand made sib-crosses and open-pollination. All insect species gave greater numbers of kernels per head than hand pollination. With $H$ debilis no significant difference was found between the efficiencies of the four insect species. Significant differences occurred between insects for $H$ annuus and H argophyllus : B terrestris (106\%) was the most efficient insect with results similar to open-pollination. Seed sets obtained with $M$ rotundata and Calliphora spp were only 77 and $73 \%$ of open pollination, respectively, but remained significantly higher than hand pollination (36\%). A mellifera (88\%) gave intermediate results between these two groups.
\end{abstract}

Helianthus / Apis mellifera / Bombus terrestris / Megachile rotundata / Calliphora spp

Résumé - Sur trois populations d'origine sauvage du genre tournesol, Helianthus annuus, Helianthus argophyllus et Helianthus debilis spp cucumerifolius, l'efficacité de la pollinisation manuelle a été comparée à celle de la pollinisation libre sur des plantes cultivées en pots (témoin) et en plein champ. Ces méthodes ont été comparées à la pollinisation en cage utilisant l'abeille Apis mellifera, le bourdon Bombus terrestris, le mégachile Megachile rotundata et des mouches bleues Calliphora spp. Quelle que soit l'espèce végétale, la méthode manuelle de croisement est toujours celle qui donne le moins de graine : 34 akènes par capitule, alors que la pollinisation libre en plein champ est la plus productive avec 150 akènes. Sur des plantes en pots, la production assurée par le bourdon (100 akènes) est comparable au témoin (95 akènes), l'abeille ( 84 akènes) présente une efficacité intermédiaire, le mégachile et les mouches bleues, avec respectivement 74 et 70 akènes, sont équivalents et les moins efficaces. On constate une interaction entre les espèces végétales et leurs pollinisateurs. Avec $H$ debilis, tous les insectes ont la même efficacité. Chez $H$ argophyllus, le bourdon et l'abeille sont supérieurs au mégachile et à la mouche. Pour $H$ annuus, la différenciation atteint quatre niveaux : le bourdon, l'abeille, le mégachile et les mouches dans l'ordre décroissant d'efficacité.

Helianthus sauvage / Apis mellifera / Bombus terrestris / Megachile rotundata / Calliphora spp

\section{INTRODUCTION}

Le genre Helianthus comprend 49 espèces dont l'importante variabilité génétique est utilisable pour l'amélioration de la régularité des performances agronomiques du tournesol en fournissant en particulier les gènes favorables pour la résistance aux maladies et l'adaptation au milieu cli-

\footnotetext{
* Correspondance et tirés à part.

Tél : (33) 0467290626 ; fax : (33) 0467293990 ; courriel : meynie@ensam.inra.fr
} 
matique (Serieys, 1980; Belhassen et al, 1992). Ces espèces sont constituées de populations qu'il faut conserver en préservant le maximum d'allèles, surtout les allèles rares, en évitant de provoquer des dérives génétiques. Elles présentent un fort degré d'autostérilité lié à des systèmes génétiques d'auto-incompatibilité sporophytique particulièrement efficaces (Heiser, 1954). La pollinisation croisée est donc de rigueur et les tournesols ont été classés parmi les plantes allogames. Le pollen ne peut être transporté par le vent que sur une courte distance : la pollinisation est essentiellement de nature entomophile. Les véritables agents pollinisateurs du tournesol sont les abeilles domestiques, les bourdons et quelques espèces d'halictes (Taséi, 1984) mais cette espèce est particulièrement attractive pour de nombreux insectes (Delaude et al, 1979).

La conservation devrait être organisée in situ mais cela relève des politiques d'aménagement de territoires et des réserves naturelles. Ce type de protection n'est pas forcément une garantie pour le maintien des formes sauvages. En effet de nombreux écotypes appartenant aux espèces Helianthus californicus (Rogers et al, 1982) et Helianthus proecox ssp hirtus (Rieseberg et Doyle, 1989) ont disparu.

Seiler et al (1990) pensent qu'un nombre de plantes trop restreint dans un environnement déficitaire en insecte pollinisateur est à l'origine de ces disparitions.

La conservation en banque est la solution de sauvegarde des populations en voie de disparition mais un autre risque de perte de la diversité génétique réside dans la gestion des reproductions. Les fécondations se font classiquement par des croisements manuels en pépinières. Cette méthode, dont le coût est élevé, donne peu de graines et contribue malgré tout à la perte de populations (Serieys, comm personnelle).

Afin de remédier à ces problèmes, certains auteurs (Collison et Wilson, 1985 ; Dozet et al, 1993) ont choisi d'exploiter des insectes en faisant reproduire les populations sauvages par des abeilles dans des cages de pollinisation. Ce mode de reproduction provoque une augmentation significative du nombre d'akènes par rapport à la pollinisation manuelle. Cependant l'efficacité de l'abeille, comparée à la pollinisation libre, n'est pas régulière. Elle obtient un gain de rendement chez des écotypes sauvages de $H$ annuus, elle occasionne un déficit pour diverses populations de l'espèce Helianthus petiolaris et elle engendre de fortes variations de production au sein de chaque groupe (Dozet et al, 1993). L'abeille n'est donc pas l'insecte pollinisateur toujours bien adapté à effectuer les reproductions en cage d'espèces sauvages d'Helianthus.

Dans le but de normaliser les productions de semences, nous avons élargi la pollinisation en cage à d'autres insectes. Deux hyménoptères appartenant à la famille des Apoidea, le bourdon et le mégachile, ainsi que des diptères de la famille des Calliphoridae, Calliphora vomitoria ou Calliphora erythrocephala ont été utilisés. Les travaux présentés ci-après proposent la mise au point de méthodes efficaces de production de semences pour trois populations d'origine sauvage d'Helianthus. Le dispositif mis en place avait pour objectif de comparer l'efficacité i) de pollinisations effectuées hors cage, en champ, en pots ou manuellement; ii) des pollinisations en cage de chaque insecte par rapport à ces méthodes; iii) de chaque insecte en fonction de l'espèce d'Helianthus. L'efficacité a été évaluée en comparant le nombre d'akènes formé par capitule, le taux de nouaison et le poids de mille grains.

\section{MATÉRIEL ET MÉTHODES}

Les expériences ont été conduites durant l'été 1992 et 1993 sur le domaine de l'UFR de génétique et amélioration des plantes de Mauguio, proche de Montpellier, et en bordure de la Méditerranée. Son climat est de type mésoméditerranéen atténué.

\section{Matériel végétal}

Les trois espèces sauvages sont originaires d'Amérique du Nord : i) $H$ annuus sauvage, $\mathrm{n}^{\circ} 376$, est un écotype particulièrement vigoureux, provenant du Nebraska, ii) $H$ argophyllus, $\mathrm{n}^{\circ} 585$, endémique de la Floride, se distingue par des feuilles d'une couleur gris argenté portant de nombreux poils soyeux, iii) $H$ debilis ssp (ucumerifolius, $\mathrm{n}^{\circ} 218$, originaire de Géorgie, est un écotype de petite taille.

Les graines ont été semées en serre au mois de mars. La levée de dormance des graines a été effectuée suivant la technique de Mouret et Piquemal, 1980. Un certain nombre de plantes ont été repiquées trois semaines plus tard dans des pots de $6 \mathrm{~L}$, puis introduit dans des cages de pollinisation $(3 \times 3 \times 2,5 \mathrm{~m})$ en polyéthylène blanc à mailles de $900 \mu \mathrm{m}$, au mois de juin lors de l'apparition des premiers capitules fleuris. Les plantes restantes ont été transplantées en champs au mois d'avril. L'irrigation des pots était réalisée quotidiennement pendant la nuit par micro asperseurs $(6,5 \mathrm{~L}$ d'eau par mètre carré). En condition de champ, 35 L d'eau par mètre carré étaient apportés une fois tous les 15 jours. 


\section{Insectes pollinisateurs}

Quatre types d'insectes pollinisateurs ont été utilisés dans cette expérimentation : i) l'abeille domestique $A$ mellifera hybride ( $A$ mellifera $\times A$ ligustica) provient de ruches faiblement peuplées, environ 2000 à 3000 ouvrières; ii) les colonies de bourdons $B$ terrestris comportant 40 à 60 ouvrières sont issues des fondatrices prélevées dans la nature au mois de mars et mises en condition artificielle d'élevage selon la technique décrite par Écalle, 1989 ; iii) les cocons de mégachile $M$ rotundata (25 cocons par cage) après un hivernage en larves au réfrigérateur subissent une incubation à $29{ }^{\circ} \mathrm{C}$ et $65 \%$ d'hygrométrie relative pendant 22 jours, selon la technique décrite par Taséi et Carré, 1984 ; iv) les larves de mouches Calliphora spp sont placées dans des bacs contenant de la sciure et incubées à $27^{\circ} \mathrm{C}$ pendant une durée de 6 à 8 j. Un apport de 2000 larves a eu lieu chaque semaine (Clements, 1982).

Les effectifs d'insectes introduits dans notre expérimentation ont été calculés en fonction des données issues des travaux de pollinisation du tournesol sauvage $H$ annuus de Wilson et Collison, 1988 pour l'abeille, de Pham-Delègue et al, 1985 sur tournesol cultivé pour l'abeille et $B$ terrestris, de Bonnet, 1991 sur carotte pour Calliphora spp. L'effectif de $M$ rotundata prend en compte son activité de butinage sur luzerne, 2000 à 4000 fleurs par jour (Taséi et Carré, 1984).

Une très forte mortalité d'abeilles ouvrières, 40 à $70 \%$ de l'effectif, a été enregistrée pendant les 2 journées qui ont suivi l'introduction des ruches dans les cages au cours des deux années. Après cette période critique, le nombre de butineuses s'est stabilisé. Le nombre d'insectes présents sur l'ensemble des capitules d'une cage au moment des comptages, qui ont eu lieu vers le début puis la fin des pollinisations, est mentionné dans le tableau I à titre indicatif. Les fortes variations proviennent des effets additifs dus aux cages, aux horaires d'observations et aux imprécisions des comptages.

\section{Dispositif expérimental}

Le dispositif expérimental réalisé en 1992 et 1993 comportait pour chacune des espèces végétales sept traitements de pollinisation avec deux répétitions. Les sept traitements étaient :
1 : pollinisation libre, plantes cultivées en pots (témoin) ;

2 : pollinisation libre, plantes cultivées en plein champ ; 3 : pollinisation manuelle, plantes cultivées en plein champ:

4 : pollinisation en cage par abeilles, plantes cultivées en pots :

5 : pollinisation en cage par bourdons, plantes cultivées en pots :

6 : pollinisation en cage par mégachiles, plantes cultivées en pots ;

7 : pollinisation en cage par mouches, plantes cultivées en pots.

Chaque parcelle comportait 24 plantes disposées en quatre rangées de six plantes espacées de $0,6 \times 0,4 \mathrm{~m}$ sous cage, $1,5 \times 1 \mathrm{~m}$ au champ. Les plantes des traitements $4,5,6$ et 7 étaient cultivées en pots et placées sous cage d'isolation. Les plantes du traitement 1 étaient cultivées en pots et placées à proximité des cages, sans filet de protection. Les plantes du traitement 2 étaient cultivées en champ sans filet de protection. La pollinisation manuelle du traitement 3 a été réalisée sur les plantes du traitement 2 en ensachant deux par deux, avant l'anthèse, les capitules appartenant à deux plantes différentes, puis en les frottant entre eux à trois reprises à 2 jours d'intervalle pendant leur floraison. Cette méthode $n$ 'a pas été reconduite sur des plantes cultivées en pots car, lors d'une étude préliminaire en 1991, on avait observé un déficit important du nombre d'akènes par rapport aux plantes cultivées en plein champ.

\section{Caractères mesurées sur les plantes}

Une fois par semaine pendant six semaines, entre le 15 juin et le 10 août de chaque année, six contrôles successifs ont été effectués pour fournir les données de production : nombre d'akènes par capitule, poids de 1000 grains et taux de nouaison. Au cours de ces dates dobservations, tous les capitules ayant atteint le stade flétrissement des ligules ont été mis sous sac, après fécondation, afin de les préserver d'un égrenage précoce et obtenir la totalité des akènes. À maturité, les capitules et les akènes ont été comptés et pesés. Au terme des pollinisations, la hauteur des plantes, le nombre de ramifications et le nombre de capitules par plante ont été observés.

Le traitement statistique de l'expérience a été réalisé suivant un plan factoriel équilibré à cinq facteurs :

Tableau I. Nombre moyen d'insectes a en activité de butinage dans une cage au cours des pollinisations.

\begin{tabular}{lcccc}
\hline & Abeille & Bourdon & Mégachile & Mouche \\
\hline Départ expérimentation & $121,5 \pm 55,9$ & $23,6 \pm 11,5$ & $15,2 \pm 5,5$ & $99,2 \pm 34,7$ \\
Fin expérimentation & $66,8 \pm 35,7$ & $10,1 \pm 5,2$ & $5,7 \pm 2,3$ & $35,8 \pm 17,1$ \\
Moyenne & $89,8 \pm 52,4$ & $16,0 \pm 10,8$ & $10,4 \pm 6,3$ & $53,7 \pm 36,9$ \\
\hline
\end{tabular}

\footnotetext{
a Nombre d'insectes posés sur l'ensemble des capitules lors d'un comptage effectué par deux personnes circulant en sens inverse entre les rangées de plantes. La durée du comptage est de $5 \mathrm{~min}$. Les moyennes sont accompagnées de l'écart type.
} 
espèces végétales, traitements, années, dates d'observations et répétitions avec respectivement $2,6,1,5$ et 1 degrés de liberté pour un total de $503 \mathrm{dL}$. Les facteurs, dates d'observations ( $F=0,92 ; P=0,47)$ et répétitions $(F=0,03 ; P=0,85)$, ont permis de vérifier la stabilité des fécondations pendant la période des contrôles et par corollaire en déduire que les effectifs d'insectes n'avaient guère évolué.

\section{RÉSULTATS}

\section{Sources de variation de la production grainière}

L'analyse statistique (tableau II) est effectuée pour le nombre d'akènes par capitule. Les principales sources de variation de la production grainière sont : en premier l'espèce végétale, en second les traitements et en troisième l'année. Les répétitions spatiales (cages ou champ) et les répétitions temporelles (dates d'observations) n'interviennent pas comme facteurs de variation. En revanche, des interactions existent entre les espèces et les traitements, les espèces et les années et enfin les espèces, les traitements et les années.

\section{Incidence des années, des méthodes, des insectes et des espèces végétales sur la production de semences}

Les moyennes du nombre d'akènes par capitule, par année, par espèce et par traitement figurent dans le tableau III.

Tableau II. Analyse générale des sources de variation pour le nombre d'akènes par capitule.

\begin{tabular}{lccccc}
\hline Sources de variation & $d l$ & $\begin{array}{c}\text { Somme } \\
\text { descarrés }\end{array}$ & $\begin{array}{c}\text { Carrés } \\
\text { moyens }\end{array}$ & $\begin{array}{c}\text { Valeur } \\
\text { de } F\end{array}$ & $\begin{array}{c}\text { Pr }>F \\
\text { Espèces }\end{array}$ \\
Traitements & 2 & 510250 & 255125 & $23,67^{\mathrm{a}}$ & 0,0001 \\
Années & 6 & 536208 & 89368 & $8,29^{\mathrm{b}}$ & 0,0001 \\
Répétitions & 1 & 36681 & 36681 & $3,40^{\mathrm{c}}$ & 0,0658 \\
Dates d'observations & 1 & 18 & 7 & 0,03 & 0,8566 \\
Espèces * traitements & 5 & 2475 & 495 & 0,92 & 0,4672 \\
Espèces * années & 12 & 198975 & 16581 & $3,12^{\mathrm{d}}$ & 0,0002 \\
Traitements* $*$ années & 2 & 39445 & 19722 & $3,72^{\mathrm{e}}$ & 0,0249 \\
Espèces * traitements * années & 6 & 42918 & 7153 & $1,35^{\mathrm{f}}$ & 0,2334 \\
Erreur & 12 & 63685 & 5307 & 9,87 & 0,0001 \\
Total & 456 & 245185 & 537 & &
\end{tabular}

a, b. c La valeur de F est obtenue en divisant la somme des carrés des écarts des interactions par la somme de leurs dl, puis en testant chaque effet au moyen de cette valeur ; d. e. ' la valeur de F est obtenue en testant chaque interaction par le carré moyen de l'interaction triple : espèces * traitements * années.

Tableau III. Nombre d'akènes par capitule et par traitement pour chaque population d'Helianthus pollinisée en 1992 et en 1993.

\begin{tabular}{|c|c|c|c|c|c|c|c|}
\hline & \multicolumn{2}{|c|}{$H$ debilis } & \multicolumn{2}{|c|}{$H$ argophyllus } & \multicolumn{2}{|c|}{$H$ annuus } & \multirow{2}{*}{$\begin{array}{l}\text { \% moyer } \\
\text { d'akènes }\end{array}$} \\
\hline & 1992 & 1993 & 1992 & 1993 & 1992 & 1993 & \\
\hline Abeille & $32,1 \pm 13$ & $48,6 \pm 15$ & $107,7 \pm 34$ & $103,7 \pm 29$ & $91,1 \pm 21$ & $120,1 \pm 26$ & 87,9 \\
\hline Bourdon & $43,0 \pm 10$ & $56,9 \pm 9$ & $118,2 \pm 24$ & $117,1 \pm 25$ & $119,9 \pm 25$ & $149,8 \pm 22$ & 105,7 \\
\hline Megachile & $29,7 \pm 12$ & $53,4 \pm 13$ & $87,1 \pm 27$ & $83,5 \pm 25$ & $82,9 \pm 20$ & $105,2 \pm 35$ & 77,1 \\
\hline Mouche & $43,4 \pm 15$ & $47,9 \pm 15$ & $72,7 \pm 25$ & $82,5 \pm 36$ & $78,5 \pm 26$ & $92,6 \pm 43$ & 72,9 \\
\hline Pol manuelle & $21,1 \pm 16$ & $21,2 \pm 16$ & $43,0 \pm 24$ & $42,7 \pm 22$ & $38,1 \pm 23$ & $39,8 \pm 23$ & 35,9 \\
\hline Pol libre en pots (T) & $44,2 \pm 10$ & $48,4 \pm 13$ & $116,5 \pm 23$ & $100,4 \pm 19$ & $115,9 \pm 14$ & $146,9 \pm 31$ & 100,0 \\
\hline Pol libre en champ & $48,7 \pm 11$ & $64,5 \pm 14$ & $153,1 \pm 21$ & $160,7 \pm 21$ & $155,8 \pm 21$ & $314,8 \pm 48$ & 156,8 \\
\hline Moyenne & \multicolumn{2}{|c|}{$43,1 \pm 18$} & \multicolumn{2}{|c|}{$99,2 \pm 41$} & \multicolumn{2}{|c|}{$117,9 \pm 70$} & - \\
\hline
\end{tabular}




\section{En fonction des années}

L'année 1992 s'est caractérisée par une diminution moyenne journalière des températures de $2,2{ }^{\circ} \mathrm{C}$ entre le 15 mai et le 15 juillet par rapport à 1993. En pollinisation libre de plein champ la production d'akènes a diminué de 25 et $50 \%$ respectivement pour les espèces $H$ debilis et $H$ annuus. En revanche, $H$ argophyllus a maintenu stable sa production mais la floraison a débuté tardivement (5 juillet) par rapport à 1993 (10 juin).

L'efficacité moindre des insectes a provoqué globalement des rendements plus faibles en 1992 qu'en 1993, particulièrement $M$ rotundata sur $H$ debilis $(-45 \%)$. Les diminutions sont de 13 , 22,30 et $33 \%$ respectivement pour les mouches, le bourdon, l'abeille domestique et le mégachile sur $H$ debilis et $H$ annuus confondus.

\section{En fonction des traitements}

Suivant les méthodes, trois résultats importants apparaissent. La méthode de pollinisation manuelle est très fortement déficitaire par rapport aux autres méthodes de pollinisation. Elle est inférieure de $50 \%$ à la pollinisation par les mouches; la méthode de pollinisation libre (plantes cultivées en plein champ) est, à l'inverse, la plus performante. Elle permet un gain de $50 \%$ par rapport au bourdon; tous les insectes engendrent de bons niveaux de production par rapport au témoin (les plantes sont cultivées en pots).

En fonction des insectes, la production d'akènes est assurée, suivant un ordre décroissant, par les bourdons dont l'efficacité est comparable à la pollinisation libre (témoin), puis par les abeilles domestiques et enfin par les mégachiles et les mouches de façon équivalente.

Les taux de nouaison ne sont pas rapportés ici car les écarts entre les différents traitements sont faibles : $26 \% \pm 3$ avec $H$ debilis et $38 \% \pm 6$ pour $H$ argophyllus et $H$ annuus. Ils différencient uniquement les bourdons des trois autres insectes pollinisateurs pour l'ensemble des espèces. Le poids de 1000 grains est resté stable quel que soit le traitement.

\section{En fonction des espèces végétales}

L'observation conjointe des espèces végétales et de leurs pollinisateurs (tableau III) confirme l'interaction observée dans le tableau II. Les écarts de production entre les insectes dépendent de l'espèce végétale pollinisée lorsque celle-ci est cultivée en pots.

Dans le cas de $H$ debilis, il n'y a pas de différence significative entre les quatre insectes, ni de différence avec le témoin pour le nombre d'akènes par capitule.

Pour $H$ argophyllus, le bourdon, l'abeille et le témoin se différencient significativement du mégachile et de la mouche.

Chez $H$ annuus sauvage, la différenciation atteint quatre niveaux : le bourdon comparable à la pollinisation libre, l'abeille, le mégachile, la mouche.

\section{Caractéristiques générales des trois populations sauvages d'Helianthus}

La floraison des trois populations sauvages s'étale généralement du mois de juin au mois de novembre. $H$ debilis (tableau IV) est une espèce très ramifiée ayant de nombreux capitules de diamètre égale ou inférieur à $0,02 \mathrm{~m}$ composés de fleurons dont la corolle présente une profondeur inférieure à $0,004 \mathrm{~m}$. $H$ annuus est haut, ses capitules ont un diamètre égal ou supérieur à $0,06 \mathrm{~m}$. La corolle de ses fleurons dépasse $0,006 \mathrm{~m}$. Les valeurs des caractéristiques des plantes de $H$ argophyllus sont comprises entre celles de $H$ debilis et celles de $H$ annuus. En revanche, sa floraison est légèrement tardive : elle débute deux à trois semaines plus tard que la floraison des deux espèces précédentes et l'étalement est plus court d'environ 5 à 6 semaines.

Ces espèces présentent une déficience reproductrice générale. Le taux de nouaison est de 26 , 46 et $54 \%$ en conditions optimales de plantes cultivées en champ, respectivement pour $H$ debilis, $H$ argophyllus et $H$ annuus. En l'absence d'insecte pollinisateur, il est égal ou inférieur à $1 \%$.

La culture en pots provoque une réduction significative des différents caractères par rapport aux plantes cultivées en plein champ. Le nombre de capitules par plante peut diminuer de $60 \%$ alors que le taux de nouaison est faiblement affecté.

\section{DISCUSSION}

Le climat défavorable de 1992 n'a pas modifié la production réalisée manuellement mais il a agit de manière différente en fonction des espèces. $H$ debilis et $H$ annuus apparaissent sensibles aux 
Tableau IV. Caractéristiques des plantes et des inflorescences des trois espèces sauvages d'Helianthus cultivées en pots, comparaison des espèces cultivées en pots en en plein champ.

\begin{tabular}{lccccc}
\hline & $\begin{array}{c}\text { H debilis } \\
\text { pots }\end{array}$ & $\begin{array}{c}\text { H argophyllus } \\
\text { pots }\end{array}$ & $\begin{array}{c}\text { H annuus } \\
\text { pots }\end{array}$ & $\begin{array}{c}\text { Moyenne } \\
\text { pots }\end{array}$ & $\begin{array}{c}\text { Moyenne } \\
\text { champ }\end{array}$ \\
\hline Hauteur des plantes $(\mathrm{m})$ & $0,92 \pm 0,19$ & $1.66 \pm 0,25$ & $1.73 \pm 0,21$ & $1.43 \pm 0,28$ & $1,70 \pm 0,37$ \\
Nombre de ramifications & $23,8 \pm 11$ & $14,6 \pm 9$ & $17,5 \pm 9$ & $16,7 \pm 12$ & $25,5 \pm 11$ \\
Nombre de capitules & $54,2 \pm 11$ & $39,3 \pm 9$ & $48,2 \pm 15$ & $47,2 \pm 12$ & $126,4 \pm 61$ \\
Nombre de fleurons & $171 \pm 29$ & $275 \pm 47$ & $319 \pm 92$ & $256 \pm 69$ & $330,8 \pm 160$ \\
Poids de 1 000 grains $(\mathrm{g})$ & $2,18 \pm 0.3$ & $8,48 \pm 0.8$ & $9,54 \pm 1,2$ & $6,88 \pm 3,1$ & $8,02 \pm 1,7$ \\
\% nouaison en pol libre & $26,9 \pm 7,7$ & $40,0 \pm 8,7$ & $40,1 \pm 7,9$ & $35,7 \pm 9,9$ & $42.0 \pm 9,1$ \\
\% nouaison hors insecte & 0,34 & 0,52 & 1,07 & - & - \\
Profondeur corolle $(\mathrm{m})^{\mathrm{h}}$ & 0,0034 & 0,0052 & 0,0061 & - & - \\
\hline
\end{tabular}

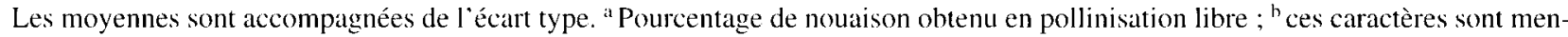
tionnés à titre indicatif car ils n'ont pas fait l'objet d'une étude systématique. Le pourcentage de nouaison hors insecte est obtenu en cage sans insecte et sans intervention manuelle.

conditions climatiques et présentent de fortes diminutions grainières. La stabilité d'H argophyllus peut s'expliquer par une floraison tardive en 1992. En effet Augé 1994 note, chez cette population par rapport aux populations précédentes, des phénomènes de compensation du rendement provoqués par la tardiveté et la durée de la floraison.

Ces trois populations présentent en outre des caractéristiques morphologiques très différentes, notamment le diamètre des capitules, le nombre de fleurons par capitule, la profondeur de la corolle. Cette forte variabilité interspécifique ainsi que des taux de nouaison extrêmement faibles, liés soit à l'absence d'insectes pollinisateurs soit à l'aptitude reproductrice de chaque espèce, renforce la nécessité de maîtriser les productions de semences.

\section{Comparaison des méthodes de production de semences}

La pollinisation manuelle, largement utilisée, est peu efficace. Pour des raisons économiques, la reproduction des populations par cette méthode est classiquement effectuée sur une base de 10 à 20 plantes. Il résulte de l'utilisation de tels effectifs une mauvaise stabilité de la variabilité intrapopulation, qui s'observe notamment par des variations de la précocité à chaque multiplication. Franklin, 1980 estime à 500 individus le nombre minimal pour maintenir le potentiel évolutif d'une plante allogame mais 100 individus préservent les allèles présents à la fréquence de $5 \%$ (Crossa, 1989). Il est donc indispensable d'augmenter le nombre de plantes à reproduire.
La pollinisation libre, pour des plantes cultivées en champ, correspond à la plus productive des méthodes. Cependant elle est difficilement utilisable à grande échelle car chaque isolement demande un minimum de 30 ha exempts de tournesol, surface indispensable à la pureté pollinique. En effet les insectes présents sur le lieu transportent du pollen étranger sur de longues distances, pouvant dépasser $2000 \mathrm{~m}$ pour l'abeille.

La troisième méthode qui consiste à faire polliniser les plantes par des insectes dans des cages d'isolement permet des productions grainières équivalentes à la pollinisation libre par des plantes cultivées en pot. Elle est déjà utilisée avec l'abeille (Dozet et al, 1993).

\section{Comparaison de l'efficacité pollinisatrice des insectes pour la production de semences}

Sur ces trois espèces d'Helianthus, le bourdon $B$ terrestris est plus efficace que l'abeille $A$ mellifera. Cette supériorité va dans le sens des observations de Pham-Delègue et al (1985) sur tournesol ou de Mesquida et al (1990) sur féverole. Cependant l'emploi d'un autre bourdon ou d'une autre abeille peut modifier ce classement. Il existe en effet une forte variabilité des aptitudes pollinisatrices inter et intraspécifique. Elle est observée entre différents Bombus (Le Guen et al, 1993) et entre des races d'abeilles et leurs hybrides (PhamDelègue et al, 1984). En revanche, Wilson et Collison (1988) ne constatent pas de différence entre quatre races d'abeilles testées sur des $H$ annuus sauvages. L'emploi du bourdon est coû- 
teux, son élevage n'étant pas encore parfaitement maîtrisé.

L'efficacité de $M$ rotundata est inférieure à celle de l'abeille domestique. À cause de l'absence constante de grains de pollen de tournesol dans les provisions des nids (Taséi, communication personnelle), cet insecte n'a pas été considéré jusqu'à présent comme pollinisateur potentiel des Helianthus. Son efficacité sur le tournesol confirme les capacités d'adaptation de cet insecte constatées par Heinrichs (1967) ou Roumet et Magnier (1993). Son élevage est techniquement simple, parfaitement maîtrisé, mais il ne butine pas aux températures inférieures à $23{ }^{\circ} \mathrm{C}$ (Taséi et Carré, 1984). Cette sensibilité aux basses températures peut expliquer le mauvais résultat observé en 1992. En revanche, cette abeille solitaire, n'ayant pas de vie sociale organisée comme l'abeille domestique ou le bourdon, peut être introduite sans contrainte dans différents milieux climatiques contrôlés.

L'utilisation de Calliphora donne les mêmes résultats que $M$ rotundata. Cet insecte, jamais testé auparavant sur des tournesols, recherche le nectar des fleurs pour assurer sa subsistance ce qui en fait un insecte pollinisateur naturel de quelques ombellifères (Ricciardelli d'Albore, 1986) notamment la carotte (Daucus carota) où il se révèle régulièrement plus efficace que l'abeille (Bonnet, 1991). Sa facilité d'élevage et sa faculté d'adaptation permettent son emploi en toute saison en différents milieux.

Les effectifs mis en place ont certainement influencé les performances des insectes. Les abeilles et les mouches sont très nombreuses par rapport aux fleurs offertes. Un capitule reçoit entre 50 et 100 visites par jour. Mesquida et al (1988. 1990) pensent qu'une surpopulation de butineuses peut provoquer soit des inhibitions de la germination des pollens par accumulation excessive de sécrétions soit des phénomènes de surexploitation des fleurs par détérioration des parties réceptrices (stigmate). À l'inverse la faible densité de mégachile peut occasionner une déficience pollinisatrice. Il faut envisager d'étudier et d'adapter le nombre d'insectes au nombre de plantes à interféconder.

\section{Interactions entre les espèces végétales et leurs pollinisateurs}

L'espèce sauvage $H$ annuus a été choisie comme témoin de référence, Wilson et Collison (1988) puis Dozet et al (1993) l'ayant utilisée comme plante modèle. $H$ argophyllus et $H$ debilis ssp cucumerifolius ont été introduits en complément pour confirmer les résultats obtenus avec $H$ annuuts. Les observations montrent que tous les insectes ne pollinisent par les trois populations sauvages avec la même efficacité. Il semble que la plante impose des limites à l'efficacité pollinisatrice des insectes. Parmi certaines caractéristiques des plantes, la profondeur de la corolle des fleurs visitées comparéee à la longueur du proboscis des insectes, peut être un facteur limitant de la fécondation (Taséi, 1976 ; Pouvreau, 1984). Ce caractère permet de classer par ordre décroissant de longueur l'abeille domestique, le bourdon, le mégachile et les mouches. La faible profondeur de la corolle de $H$ debilis permet l'accès aux nectaires par tous les insectes. La corolle d'H annuus, plus profonde, limite l'accès des fleurons femelles aux abeilles et aux bourdons.

Toutefois cette hypothèse n'explique pas la supériorité du bourdon vis-à-vis de l'abeille dans le cas d' $H$ annutus. Une autre explication possible est que le bourdon est un insecte rapide : il visite deux fois plus de fleurs que l'abeille par unité de temps (Delaude et Rollier, 1977) restant deux fois moins longtemps sur un capitule (Pham-Delègue et al, 1985). Cette rapidité peut favoriser la dispersion pollinique et limiter l'assèchement des stigmates.

\section{Conclusion}

Dans un programme de multiplication d'espèces sauvages du genre Helianthus, le gestionnaire doit rejeter la méthode manuelle de pollinisation, trop peu productive, exigeante en personnel et mal adaptée au maintien de la variabilité. La pollinisation libre implique par contre trop de contraintes d'isolement.

La pollinisation dirigée en cage s'affranchit des inconvénients majeurs liés à ces deux méthodes. La technique de la culture en pot diminue la hauteur et le nombre de ramifications favorisant l'augmentation de la densité. Elle offre aussi l'avantage d'adapter les substrats et les milieux aux caractéristiques agrophysiologiques des espèces calcifuges et calcicoles qui présentent des sensibilités différentes à la photopériode et à la température selon Rogers et al (1982).

En disposant de plusieurs insectes, complémentaires en raison de leurs facultés d'adaptation à différents milieux, à différents climats et à différentes espèces, les programmes de reproduction pourront être organisés en fonction des caractéris- 
tiques intrinsèques des diverses formes sauvages du genre Helianthus et des aptitudes des insectes. En effet, la floraison des populations sauvages peut débuter tôt, au mois de mai, ou très tard, en décembre et janvier (Serieys, 1992). Dans ces conditions extrêmes de fécondation les mouches bleues et les mégachiles présentent quatre avantages majeurs par rapport aux abeilles et aux bourdons : ils sont disponibles toute l'année, l'élevage peut être réalisé par l'utilisateur, les coûts sont très faibles et ils s'emploient indifféremment dans de petites ou de grandes structures, en phytotron, en serre et en champ.

\section{REMERCIEMENTS}

Nous remercions $\mathrm{H}$ Serieys (Inra, UFR-Gap, Montpellier) pour avoir choisi et fourni les espèces sauvages d'Helianthus puis prodigué ses conseils tout au long des essais, JN Taséi (Inra, Zoologie, Lusignan) pour les cocons de $M$ rotundata, les techniques et le matériel d'élevage ainsi que pour ses enrichissantes critiques concernant le manuscrit, C Écalle (Inra, SAPF, Lusignan) pour les fondatrices de $B$ terrestris et ses directives d'élevage.

\section{RÉFÉRENCES}

Augé G (1994) Analyse de la diversité morphologique et isoenzymatique de populations artificielles d'Helianthus annuels : conséquences pour une gestion des ressources génétiques adaptée au Tournesol (H annuus var macrocarpus L). Thèse, Institut national agronomique Paris-Grignon

Belhassen E, Augé G, Vares D (1992) The use of population genetics in Sunflower resource managment. Proceeding of the 13 th International Sunflower Conference. 7-11 Septembre, Pise, Italie.

Bonnet A (1991) Production and quality of carrots seeds. In : Eucarpia Carrot 91. Proceedings of the IVth meeting on breeding of Carrots, AvignonMontfavet, 18-20 juin, 131-145

Clements R (1982) Blowflies worth considering for tunnel pollination. Grower 97, 22, 24-26

Collison VL, Wilson RL (1985) Comparaison of honey bees with hand and self pollination for obtaining seed from wild type sunflowers in cages. Southwestern Entomologist 10, 268-273

Crossa J (1989) Methodologies for estimating the sample size required for genetic conservation of outbreeding crops. Theor Appl Genet 77, 153-161

Delaude A, Rollier M (1977) Pollinisation et modalités de production des semences hybrides de tournesol. Cetiom Inform Tech 56, 15-24
Delaude A, Taséi JN, Rollier M (1979) Pollinator insects of sunflower (Helianthus annuus $\mathrm{L}$ ) in France. Pollination of male sterile lines for hybrid production. Proceedings of the IVth Int Symp on Pollination. College Park, 1978, 29-40

Dozet BM, Mandelc S, Skoric D, Farkas B (1993) Use of honey bees for controlled interpollination of wild $H$ annuts $\mathrm{L}$ and $H$ petiolaris Nuttall. Helia 16,19 , 69-76

Écalle C (1989) Technique d'élevage et utilisation des bourdons (Bombus Latreille) à partir des reines capturées dans la nature. Notice technique, Station d’amélioration des plantes fourragères, InraLusignan

Franklin IR (1980) Evolutionary change in small population. In : Conservation Biology: An EvolutionaryEcological Perspective (ME Soulé, BA Wilcox, Sunderland, eds), Mass, Sinauer, 135-149

Heinrichs DH (1967) Seed increase of Alfalfa in growth chambers with Megachile rotundata F. Can $J$ Plant Sci 47, 691-694

Heiser CB (1954) Variation and subspeciation in common sunflower (Helianthus anmuus). Am Midl Naturalist 51 . 287-305

Le Guen J, Mesquida J, Pierre JS, Morin G, Taséi JN, Carré S (1993) Efficacité pollinisatrice de différents traitements sur deux lignées de féverole de printemps (Vicia faba L var equina Steudel), à des niveaux d'autofertilité différents, avec utilisation de diverses espèces de Bombus Latr (Hymenoptera, Apidae). Apidologie 24, 129-145

Mesquida J, Renard M, Pierre IS (1988) Rapeseed (Brassica napus L) productivity: the effect of honeybees (Apis Mellifera L) and different pollination conditions in cage and field tests. Apiclologie $19,1,51-72$

Mesquida J, Le Guen J, Taséi JN, Carré S, Morin G (1990) Modalités de la pollinisation chez deux lignées de féverole de printemps (Vicia faba L var equina Steudel). Effets sur les coulures, la productivité et les taux de croisements. Apidologie 21, 511525

Mouret JC, Piquemal G (1980) L'utilisation de l'éthéphon (acide 2 éthanephosphonique) pour lever la dormance des graines de tournesol (Helianthus annuus L). IX Conférence Intern del Girasol, Torremolinos, 8-13 juin, Malaga, Espagne

Pham-Delègue MH, Masson C, Douault P (1984) Étude comparée, effectuée au laboratoire, des aptitudes au butinage d'abeilles de race Apis mellifera ligustica et d'hybrides interraciaux Apis mellifera (ligustica $\times$ calacasica) $\times$ mellifera. Apidologie 15, 1, 33-42

Pham-Delègue MH. Fonta C, Masson C, Douault P (1985) Étude comparée du comportement de butinage d'insectes pollinisateurs (abeilles domestiques Apis mellifera $\mathrm{L}$ et bourdons Bombus terrestris L) sur des lignées parentales d'hybrides de tournesol Helianthus annuus L. Acta Oecologica, Oecol Applic 6, 47-67 
Pouvreau A (1984) Biologie et écologie des bourdons. In : Pollinisation et productions végétales (Pesson et Louveaux, eds), Inra, Paris, 565-629

Ricciardelli d'Albore GC (1986) Les insectes pollinisateurs de quelques ombellifères d'intérêt agricole et condimentaire (Angelica archangelica L, Carum carni L, Petroselinum crispum AW Hill, Apium graveolens $\mathrm{L}$, Pimpinella anisum $\mathrm{L}$, Daucus carota $\mathrm{L}$, Foeniculum vulgare MILLER V, Azoricum THELL). Apidologie 17, 2, 107-124

Rieseberg LH, Doyle MF (1989) Allozyme variation in Helianthus praecox ssp hirthus, a rare sunflower from southern texas. Aliso 12, 2, 379-386

Rogers CE, Thompson TE, Seiler GJ (1982) Sunflower species of the United States. Pub Nat Sunflower Ass, Fargo (ND), États-Unis, $75 \mathrm{p}$

Roumet P, Magnier I (1993) Estimation of hybrid seed production and efficient pollen flow using insect pollination of male sterile soybeans in caged plots. Euphytica 70, 61-67

Seiler GJ, Pomeroy JS, Dozet B, Gravilova V (1990) Wild sunflower germplasm collected from the Great Lakes Region of the United States. Helia 13, 21-27
Serieys H (1980) Utilisation des espèces sauvages d'Helianthus pour l'amélioration du tournesol cultivé. Proceedings of the IXth International Sunflower Conference, Torremolinos, Spain, 107-121

Serieys H (1992) Ressources phytogénétiques Tournesol. Catalogue des espèces sauvages du genre Helianthus. Ensa-Inra, station de génétique et amélioration des plantes de Montpellier, $412 \mathrm{p}$

Taséi JN (1976) Les insectes pollinisateurs de la féverole d'hiver (Vicia faba equina $\mathrm{L}$ ) et la pollinisation des plantes mâle-stériles en production de semence hybride. Apidologie 7, 1, 1-38

Taséi JN (1984) Cultures à graines oléagineuses des régions tempérées. In : Pollinisation et productions végétales (Pesson et J Louveaux, eds), Inra, Paris, 309-330

Taséi JN, Carré S (1984) Élevage des mégachiles en France. Notice technique, laboratoire de zoologie, Inra, Lusignan

Wilson RI, Collison VL (1988) Field cage study of the effects of four honey bee stains and hand pollination of the seed of wild sunflower. Seed Sci Technol 16, $471-475$ 